\title{
Frugivoria e especificidade por hospedeiros na erva-de-passarinho Phoradendron rubrum (L.) Griseb. (Viscaceae) ${ }^{1}$
}

\author{
ELIANA CAZETTA ${ }^{2,3}$ e MAURO GALETTI ${ }^{2}$
}

(recebido: 16 de fevereiro de 2006; aceito: 24 de maio de 2007)

\begin{abstract}
Frugivory and host specificity in the mistletoe Phoradendron rubrum (L.) Griseb. (Viscaceae)). We investigated the behavior of avian seed disperser and host characteristics that influence the presence and abundance of the mistletoe Phoradendron rubrum (Viscaceae) on Floresta Estadual Edmundo Navarro de Andrade, Rio Claro, São Paulo - Brazil. During 90 hours of focal observation we recorded 280 bird visits, Euphonia chlorotica and E. cyanocephala were the main seed disperser, responsible for 98,21\% of the visits. Phoradendron rubrum parasitized two tree species: Tabebuia ochracea (Cham.) Standl. (Bignoniaceae) and Melia azedarach L. (Meliaceae). The location in the canopy and the size of the branch affected the presence of mistletoes. The frequency of $P$. rubrum was higher in the upper canopy and in branches between 1.1 and $2.0 \mathrm{~cm}$ in diameter. Characteristics related to the host size (height, DBH and canopy diameter) also affect the number of infection. Mistletoe presence and abundance can be influenced by disperser behavior and characteristics of host plants.
\end{abstract}

Key words - Euphonia, frugivory, host characteristics, mistletoes, parasites

RESUMO -(Frugivoria e especificidade por hospedeiros na erva-de-passarinho Phoradendron rubrum (L.) Griseb. (Viscaceae)). Investigou-se o comportamento das aves dispersoras e as características individuais dos hospedeiros que podem afetar a presença e abundância das ervas-de-passarinho Phoradendron rubrum (Viscaceae) na Floresta Estadual Edmundo Navarro de Andrade, Rio Claro, São Paulo - Brasil. Durante 90 horas de observações focais foram registradas 280 visitas de aves, sendo Euphonia chlorotica e E. cyanocephala os principais dispersores, responsáveis por 98,21\% das visitas. Phoradendron rubrum foi observado parasitando apenas duas espécies de árvores: Tabebuia ochracea (Cham.) Standl. (Bignoniaceae) e Melia azedarach L. (Meliaceae). A localização na copa do hospedeiro e o tamanho do galho de inserção afetam a presença de infestações. As ervas-de-passarinho são encontradas principalmente no estrato superior da copa e em classes de diâmetro pequenas (entre 1,1 e 2,0 cm). As características relacionadas ao tamanho do hospedeiro (altura, DAP e diâmetro da copa) também estão relacionadas com o número de infestações. A presença e abundância das ervas-de-passarinho pode ser influenciada pelo comportamento dos dispersores e pelas características dos hospedeiros.

Palavras-chave - características dos hospedeiros, ervas-de-passarinho, Euphonia, frugivoria, parasitas

\section{Introdução}

As ervas-de-passarinho são plantas hemiparasitas que crescem em galhos de árvores ou arbustos hospedeiros, estabelecendo uma conexão com o xilema deste para retirar água e alguns nutrientes (Kuijt 1969). A grande maioria das espécies de erva-de-passarinho depende das aves para a dispersão de suas sementes, sendo algumas destas altamente especializadas no consumo dos seus frutos (Reid et al. 1995).

Para que seu estabelecimento ocorra, é necessário que as sementes germinem sobre o galho, e o haustório precisa penetrar no hospedeiro desenvolvendo uma união complexa com o tecido vascular deste (Kuijt 1969,

\footnotetext{
1. Parte do trabalho de conclusão de curso da primeira autora para obtenção do grau de Bacharel em Ciências Biológicas, Universidade Estadual Paulista, Rio Claro.

2. Universidade Estadual Paulista, Departamento de Ecologia, Grupo de Fenologia e Dispersão de Sementes, Caixa Postal 199, 13506-900 Rio Claro, SP, Brasil.

3. Autor para correspondência: elianac@rc.unesp.br
}

Tennakoon \& Patê 1996). Sendo assim, a dispersão de sementes é particularmente um evento crítico, uma vez que as sementes precisam ser depositadas em galhos hospedeiros suscetíveis para que haja alguma chance de estabelecimento (Monteiro et al. 1992, Sargent 1995). A relação com os hospedeiros é um fator limitante, uma vez que fatores como viabilidade, qualidade, resistência do hospedeiro ao parasita e preferência do parasita são fundamentais (Marvier \& Smith 1997). Portanto, a germinação e o estabelecimento dependem do sucesso na dispersão de sementes juntamente com a compatibilidade com o hospedeiro.

Algumas ervas-de-passarinho são generalistas, podendo parasitar uma variedade de hospedeiros, enquanto outras apresentam uma limitação na quantidade de hospedeiros que parasitam, sendo que algumas poucas espécies são conhecidas por parasitarem apenas uma única espécie de planta (Norton \& Carpenter 1998). Apesar de poderem parasitar uma diversidade de hospedeiros, mesmo as ervas-de-passarinho generalistas apresentam preferências e parasitam alguns 
hospedeiros mais freqüentemente do que outros (Norton \& Carpenter 1998).

A germinação das ervas-de-passarinho é pouco influenciada pelo substrato ao qual a semente está aderida (Radomiljac 1998). No entanto, o diâmetro do galho exerce um forte efeito na persistência e no estabelecimento das sementes (Reid 1989, Sargent 1995, Yan \& Reid 1995, Ladley \& Kelly 1996, Norton \& Ladley 1998). Outras características dos hospedeiros também são importantes, sendo a idade e o tamanho destes importantes para o prevalecimento das ervasde-passarinho (Martínez-del-Rio et al. 1995, Bannister \& Strong 2001).

O gênero Phoradendron é conhecido por parasitar uma única espécie de hospedeiro em determinadas áreas (Clay et al. 1985). Na Floresta Estadual Edmundo Navarro de Andrade, P. rubrum ocorre em duas espécies hospedeiras: Tabebuia ochracea e Melia azedarach, no entanto, os fatores que determinam o parasitismo não são conhecidos. Devido a diferenças no grau de ocorrência das infestações entre os hospedeiros, acreditamos que além do comportamento das aves, características individuais dos hospedeiros podem desempenhar um papel importante na ocorrência e freqüência das infestações. Diante disso, os objetivos deste trabalho foram determinar as espécies de aves envolvidas na dispersão de sementes e avaliar as características dos hospedeiros que poderiam influenciar a presença e abundância de P. rubrum.

\section{Material e métodos}

Área de estudo - O estudo foi realizado na Floresta Estadual Edmundo Navarro de Andrade ( $22^{\circ} 26^{\prime} \mathrm{S}$ e $47^{\circ} 31^{\prime} \mathrm{W}$ ), situado na área urbana do município de Rio Claro, região central do Estado de São Paulo, Brasil. A Floresta Estadual ocupa atualmente uma área de 2.314 ha, cuja cobertura vegetal é dominada por reflorestamento de Eucalyptus spp., mas também ocorre áreas com mata secundária em estágio sucessional (Moura 1998). Existe um arboreto implantado em 1944 com cerca de 17 ha contendo 1.222 exemplares de árvores diversas e 52,41 ha de plantio de coníferas (Moura 1998). Espécie estudada - Phoradendron rubrum (L.) Griseb. (Viscaceae) apresenta uma inflorescência em espiga, as flores são pequenas, os frutos são pseudobagas, pequenas e de coloração laranja, pesando em média $0,044 \mathrm{~g} \pm 0,006$, com aproximadamente $2,33 \mathrm{~mm} \pm 0,3 \mathrm{~mm}$ de comprimento e 1,65 $\mathrm{mm} \pm 0,3 \mathrm{~mm}$ de largura $(n=30)$. Os frutos possuem um exocarpo duro que é descartado pelas aves. As sementes apresentam um endosperma carnoso e dois cotilédones. Phoradendron rubrum apresenta sincronia na floração e frutificação, sendo que a floração ocorre no período de outubro a janeiro, os frutos imaturos de dezembro a março e os frutos maduros de março a junho. $\mathrm{Na}$ área de estudo $P$. rubrum infecta apenas uma espécie de ipê, Tabebuia ochracea (Bignoniaceae) que foi plantada na área de estudo e uma espécie exótica, erva-de-santa-bárbara, Melia azedarach (Meliaceae).

Frugivoria - Realizamos observações focais (ver Galetti et al.2002) no período de maio a junho de 1999 , abril a junho de 2000 e março a abril de 2001 em cinco indivíduos de ipê, Tabebuia ochracea (Bignoniaceae), e em cinco indivíduos de erva-de-santa-bárbara, Melia azedarach (Meliaceae), infectados por Phoradendron rubrum. Durante as sessões de observações, que constituíam de períodos de quatro a cinco horas entre $6 \mathrm{~h}$ e $11 \mathrm{~h}$ da manhã, foram registradas as espécies de aves visitantes, o número de visitas de cada espécie, o tempo de permanência na planta e o número de frutos consumidos (Pizo 1997, Galetti et al. 2002). Consideramos visita apenas quando houve consumo de frutos, e a nossa unidade amostral foi o hospedeiro e não um único indivíduo de P. rubrum. O consumo de frutos e os tempos totais de permanência foram determinados a partir dos dados de observações completas, nos quais as aves puderam ser observadas durante todo o período da visita. Germinação - Testes de germinação foram conduzidos em casa de vegetação, onde foram comparados os seguintes tratamentos: 1) frutos com exocarpo retirado manualmente, 2) frutos com exocarpo intacto, 3) frutos que passaram pelo tubo digestivo de Euphonia spp. e 4) Chiroxiphia caudata. As sementes defecadas por Euphonia spp. foram coletadas no campo e as sementes defecadas por Chiroxiphia caudata foram coletadas em cativeiro. Utilizamos 100 sementes para cada tratamento, que foram colocadas para germinar sobre papel de filtro em placas de Petri, e foram observadas e regadas semanalmente por noventa dias, prazo após o qual todas as sementes germinaram ou tornaram-se inviáveis. A germinação foi considerada a partir do aparecimento da radícula.

Características dos hospedeiros - Selecionamos uma área de 21 ha composta por um mosaico entre o arboreto e a mata secundária onde todos os indivíduos de Tabebuia ochracea e Melia azedarach, contendo ou não infestações, foram marcados e identificados. Em todos os hospedeiros infectados, o número total de ervas-de-passarinho foi registrado, sendo anotado se a infestação estava no estrato inferior, médio ou superior da copa do hospedeiro. A copa do hospedeiro foi dividida horizontalmente em três partes iguais para definição do estrato que a erva-de-passarinho ocupava (Norton et al. 1997).

Para avaliar em quais classes de diâmetro as ervas-depassarinho se estabelecem, utilizamos cinco indivíduos de Tabebuia ochracea e cinco indivíduos de Melia azedarach selecionados aleatoriamente. Os diâmetros dos galhos nos quais as ervas-de-passarinho estavam inseridas foi registrado com o auxílio de uma fita métrica.

Para determinar se as características individuais dos hospedeiros influenciam a freqüência e ocorrência das 
infestações, para todos os hospedeiros de $P$. rubrum registramos a altura, o diâmetro na altura do peito (DAP), o fuste, o diâmetro da copa e a distância de outro hospedeiro mais próximo (Martínez-del-Rio et al. 1995). A copa do hospedeiro foi projetada no solo e o com o auxílio de uma fita métrica o diâmetro da mesma foi obtido. A altura do hospedeiro foi calculada com o auxílio de um clinômetro e medida da distância do observador até a planta, utilizando a fórmula: $\operatorname{Tg} \alpha=H / D$, onde: $\alpha$ é o ângulo, $\mathrm{H}$ é a altura a ser calculada e D é a distância do observador a planta.

Análise dos dados - Comparamos o número de infestações entre os hospedeiros de $P$. rubrum através do teste $U$ de Mann Whitney. Para avaliarmos em qual estrato da copa do hospedeiro as ervas-de-passarinho ocorrem em maior intensidade utilizamos o teste qui-quadrado. A presença de infestações entre os indivíduos de Melia azedarach foi relacionada com o tamanho do hospedeiro através de uma regressão logística. Para as análises de correlação foi aplicado o coeficiente de correlação de Spearman entre as características dos hospedeiro e o número de infestações, sendo considerado para a análise apenas os dados referentes a Melia azedarach, devido ao baixo número de Tabebuia ochracea encontrado. Todos os procedimentos estatísticos seguiram Zar(1996).

\section{Resultados}

Frugivoria - Durante 90 horas de observações focais em Phoradendron rubrum, registramos 280 visitas de aves, num total de seis espécies, pertencentes a três famílias diferentes (tabela 1). Embora todas as espécies possam ser consideradas dispersoras, pois todas engoliram os frutos inteiros e não pareceram destruir as sementes com o bico, Euphonia chlorotica e $E$. cyanocephala podem ser considerados os principais dispersores de P. rubrum na área de estudo, uma vez que foram responsáveis por $98,21 \%$ das visitas. As duas espécies foram agrupadas nas análises porque somente os indivíduos machos podem ser distinguidos em campo, sendo as fêmeas e os juvenis de ambas as espécies muito semelhantes entre si. As duas espécies de Euphonia permaneceram 4,64 $\pm 15,4$ minutos por visita (média e desvio padrão) e alimentaram-se de cerca de $10,83 \pm 6,89$ frutos por minuto (média e desvio padrão). As outras espécies visitaram $P$. rubrum poucas vezes e consumiram apenas alguns frutos (tabela 1).

Euphonia spp. ao alimentar-se dos frutos de $P$. rubrum ingere a polpa e a semente, deixando cair o exocarpo. Sempre defecam as sementes nos galhos e algumas vezes estas caem ao solo. As sementes são defecadas na forma de um "colar de contas", que esticam e se fixam ao galho devido ao visco que envolve as sementes (Restrepo 1987).

Germinação - As sementes de P. rubrum em que o exocarpo foi retirado manualmente e que passaram pelo tubo digestivo das aves apresentaram altas porcentagens de germinação (tabela 2). Quando comparamos as sementes que passaram pelo tubo digestivo de Euphonia sp. com as sementes que passaram pelo tubo digestivo de Chiroxiphia caudata não encontramos diferença significativa $\left(\chi^{2}=0,19, g l=1, P=0,66\right)$. As sementes em que o exocarpo foi retirado manualmente também não diferiram estatisticamente das sementes que passaram pelo tubo digestivo das aves $\left(\chi^{2}=0,25, g l=\right.$ $1, P=0,88)$. Nenhuma das sementes que permaneceu com o exocarpo intacto germinou (tabela 2 ).

Características dos hospedeiros - Um total de 37,33\% $(n=75)$ das espécies hospedeiras de P. rubrum encontravam-se infectadas. O número total de infestações foi de 171, sendo que infestações isoladas foram encontradas em apenas 6 árvores e o maior número de ervas-de-passarinho em uma única árvore

Tabela 1. Espécies de aves que consumiram Phoradendron rubrum em 90 horas de observações focais na Floresta Estadual Edmundo Navarro de Andrade, Rio Claro, SP ( $n=$ número de observações com medidas completas).

Table 1. Bird species consuming Phoradendron rubrum in 90 hours of focal observations at the Floresta Estadual Edmundo Navarro de Andrade, Rio Claro, SP ( $n=$ number of observations with complete measurements).

\begin{tabular}{lcccc}
\hline Espécie & N. de visitas & \% de visitas & $\begin{array}{c}\text { Consumo frutos/min. } \\
\text { (média } \pm \text { desvio padrão }(n))\end{array}$ & Permanência/min. \\
\hline Thraupinae & 275 & 98,21 & $10,83 \pm 6,89(47)$ & $4,64 \pm 15,40$ \\
$\quad$ Euphonia spp. & 2 & 0,71 & $2(1)$ & 3 \\
$\quad \begin{array}{l}\text { Thraupis sayaca } \\
\text { Tangara cayana }\end{array}$ & 1 & 0,36 & - & 4 \\
$\begin{array}{l}\text { Mimidae } \\
\quad \text { Mimus saturninus }\end{array}$ & 1 & 0,36 & - & - \\
$\begin{array}{l}\text { Tyrannidae } \\
\text { Elaenia } \text { sp. }\end{array}$ & 1 & 0,36 & $9(1)$ & 31 \\
\hline
\end{tabular}


foi 37. Todos os indivíduos de Tabebuia ochraceae encontrados estavam infectados e embora a prevalência de infestações entre os indivíduos de Melia azedarach seja maior (37\% das árvores na área de $21 \mathrm{ha}$ ), Tabebuia ochracea apresentou maior intensidade de infestação, sendo esta diferença estatisticamente significativa (Mann Witney, $U=14,21, P=0,0002$ ) (tabela 3).

A maioria das ervas-de-passarinho estão localizadas no estrato superior da copa dos hospedeiros $(\mathrm{N}=115$; $67,25 \%)$, seguidas pelo estrato médio $(\mathrm{N}=50 ; 29,25 \%)$ e posteriormente pelo estrato inferior $(\mathrm{N}=6 ; 3,5 \%)$. Existe diferença significativa entre o estrato da copa que $P$. rubrum ocupa nos hospedeiros, sendo o número de infestações significativamente maior no estrato superior $\left(\chi^{2}=61,30, g l=3, P<0,0001\right)$. A maioria das infestações $(55,85 \%)$ ocorre nas classes de diâmetros dos galhos hospedeiros entre 1,1 e 2,0 cm, praticamente não sendo encontradas infestações na classe inferior a esta e em galhos com mais de 6,0 cm de diâmetro (Figura 1).

Para os indivíduos de Melia azedarach, a presença ou não de infestações está relacionada com a altura dos hospedeiros $\left(\chi^{2}=7,59, g l=1, P=0,005\right)$ e o número de infestações apresentou correlação significativa com a altura do hospedeiro $\left(\mathrm{r}_{\mathrm{s}}=0,26, n=70, P=0,02\right)$, o DAP $\left(\mathrm{r}_{\mathrm{s}}=0,49, n=70, P<0,0001\right)$, o diâmetro da copa $\left(\mathrm{r}_{\mathrm{s}}=0,23, n=70, P=0,05\right)$ e a distância do hospedeiro mais próximo $\left(\mathrm{r}_{\mathrm{s}}=0,28, n=70, P=0,01\right)$.

\section{Discussão}

Na área de estudo são encontradas 255 espécies de aves, das quais cerca de $20 \%$ são frugívoras e poderiam alimentar-se dos frutos de P. rubrum (Willis 2003). No entanto, apenas cinco espécies de aves consumiram os frutos de P. rubrum na área de estudo e Euphonia chlorotica e E. cyanocephala foram responsáveis pela quase totalidade das visitas. O gaturamo-rei (Euphonia cyanocephala) é um pássaro de áreas abertas e semi-abertas, não associado inteiramente a florestas (Ridley \& Tudor 1989). Na região de Campinas, ocorre como um migrante de inverno, aparecendo no período entre abril e junho, e está intimamente associado à frutificação de P. rubrum, recurso compartilhado com o congênere Euphonia chlorotica (Lima \& Aleixo dados não publicados). Nos Neotrópicos poucos são os trabalhos realizados com ervas-de-passarinho, porém outros estudos também constataram que espécies do gênero Euphonia são os principais dispersores de sementes (Motta Júnior 1991,
Argel-de-Oliveira 1999, Gondim 2001). A família Viscaceae apresenta um grupo homogêneo de dispersores de sementes, a grande maioria da Subordem Oscine, o que inclui Thraupinae e Euphonia/Chlorophonia (Restrepo et al. 2002).

As aves do gênero Euphonia, assim como as Clorophonia, que comumente alimentam-se de ervasde-passarinho, apresentam um tubo digestivo modificado, onde está ausente um estômago muscular e uma moela (Forbes 1880, Jordano 1992). Segundo Snow \& Snow (1971) esta característica está associada ao hábito dessas aves de alimentarem-se de epífitas em geral. Embora esta característica não influenciou na qualidade da germinação das sementes, Euphonia spp. é essencial para a exposição do visco, que é necessário para a fixação da semente no galho da planta hospedeira. Reid (1989) e Murphy et al. (1993) também não encontraram

Tabela 2. Porcentagem de germinação das sementes de Phoradendron rubrum nos diferentes tratamentos. Controle = sementes em que o exocarpo foi removido manualmente.

Table 2. Germination percentage of Phoradendron rubrum seeds in different treatments $($ Control $=$ seeds with hand removed exocarp).

\begin{tabular}{lcc}
\hline \multicolumn{1}{c}{ Tratamento } & N. sementes & \% germinação \\
\hline Controle & 100 & 94 \\
Euphonia spp. & 100 & 94 \\
Chiroxiphia caudata & 100 & 94 \\
Sementes c/ exocarpo & 100 & 0 \\
\hline
\end{tabular}

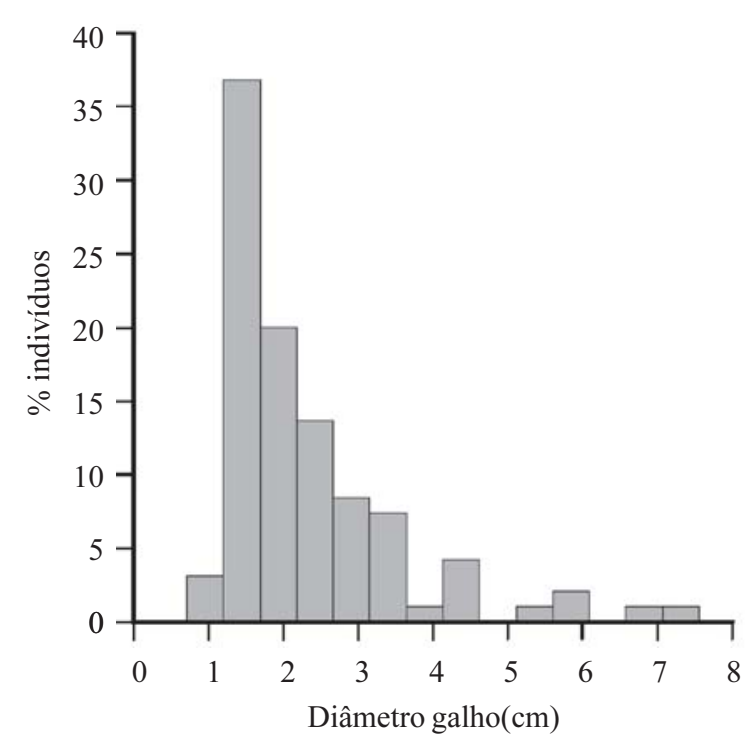

Figura 1. Número de infestações de Phoradendron rubrum em diferentes classes de diâmetros do galho hospedeiro.

Figure 1. Number of Phoradendron rubrum infestation in different size of host branch diameter. 
diferenças na germinação das sementes de ervas-depassarinho consumidas por aves com tubo digestivo modificado e não modificado. No entanto, Murphy et al. (1993), constatou que após cinco meses as sementes que passaram por aves com tubo digestivo modificado obtiveram uma maior porcentagem de estabelecimento.

A ocorrência de Phoradendron rubrum principalmente no estrato superior da copa dos hospedeiros pode ser devido a maior incidência de luz neste estrato, o que o torna mais favorável a fotossíntese, possibilita um ambiente que pode permitir a abertura dos estômatos, maximizando a transpiração e assegurando um gradiente de água entre a erva-depassarinho e o hospedeiro (Ullmann et al. 1985). Embora não tenha sido quantificado, pode ser que as ervas-de-passarinho ocorram em maior quantidade no estrato superior simplesmente porque neste estrato o número de galhos de menor diâmetro é maior, possibilitando um maior número de poleiros acessíveis aos dispersores.

Características de poleiros selecionados pelas aves dentro das espécies hospedeiras podem influenciar os padrões de recrutamento, sendo que o tamanho do galho no qual a semente é depositada influencia muito o destino das sementes (Sargent 1995). Na área de estudo a maioria das ervas-de-passarinho foram encontradas na classe de diâmetro que variou entre 1,1 e $2,0 \mathrm{~cm}$. Sargent (1995) ao estudar Phoradendron robustissimum encontrou um resultado bastante semelhante, em que o maior estabelecimento foi encontrado entre 1,0 e 1,4 $\mathrm{cm}$. As classes ainda menores de diâmetros apresentam menor quantidade de infestações devido à alta mortalidade destes galhos hospedeiros e as classes maiores de diâmetros também são pouco infectadas provavelmente devido à incapacidade de penetração do haustório no xilema do hospedeiro.

Um fator muito importante a ser considerado é o tamanho das espécies dispersoras. O macho de Euphonia chlorotica mede aproximadamante $9,5 \mathrm{~cm}$ e pesa 8,8 g, Euphonia cyanocephala mede aproximadamente $11 \mathrm{~cm}$
(Sick 1997). O pequeno tamanho destas aves deve ser considerado o principal fator na seleção de galhos menores. Reid (1991) sugere que o requerimento por galhos menores pode ter promovido uma coevolução entre aves com massa corporal pequena e as ervas-de-passarinho.

As características relacionadas com o tamanho dos hospedeiros influenciam na presença e abundância de P. rubrum na Floresta Estadual Edmundo Navarro de Andrade. Outros trabalhos também encontraram relação entre a altura e o diâmetro dos hospedeiros com a intensidade de infestação (Downey et al. 1997, Bannister \& Strong 2001). Portanto, essas diferenças individuais entre os hospedeiros são fatores fundamentais para explicar a abundância e distribuição das ervas-de-passarinho (López-de-Buen \& Ornelas 2002).

Overton (1994) utilizou modelos matemáticos para identificar as condições necessárias para o prevalência do parasita com o aumento da idade do hospedeiro. A baixa probabilidade de uma infestação inicial e a baixa probabilidade da perda de uma infestação existente leva a um aumento na proporção de hospedeiros infectados entre os indivíduos mais velhos. Em alguns casos a altura e o diâmetro da árvore podem ser indicativos da idade da planta, o que poderia explicar o maior número de infestações em plantas maiores. Uma outra hipótese seria que as espécies de maior tamanho, com copas maiores, estão proporcionando uma maior quantidade de sítios propícios para a germinação e estabelecimento das ervas-de-passarinho. No entanto, um fator fundamental a ser considerado é que as aves dispersoras podem apresentar preferências por utilizar árvores maiores como poleiro ou sítios de alimentação (Aukema \& Martínez-del-Rio 2002).

$\mathrm{O}$ fato das aves dispersoras percorrerem pequenas distâncias após alimentarem-se dos frutos pode levar a dispersão das sementes sobre as plantas parentais ou em árvores vizinhas (Overton 1996). A distribuição agrupada entre as ervas-de-passarinho tem sido observada em muitos estudos (Monteiro et al. 1992, Overton 1994, Martínez-del-Rio et al. 1995, Aukema \& Martínez-del-

Tabela 3. Total de hospedeiros, hospedeiros com infestações e número de Phoradendron rubrum por hospedeiro na Floresta Estadual Edmundo Navarro de Andrade, Rio Claro, SP.

Table 3. Total number of host, infected hosts and number of Phoradendron rubrum by host at the Floresta Estadual Edmundo Navarro de Andrade, Rio Claro, SP.

\begin{tabular}{lcccc}
\hline \multicolumn{1}{c}{ Hospedeiros } & $\begin{array}{c}\text { N. de } \\
\text { hospedeiros }\end{array}$ & $\begin{array}{c}\text { N. hospedeiros } \\
\text { infectados }(\%)\end{array}$ & $\begin{array}{c}\text { N. de } \\
\text { infestações }\end{array}$ & $\begin{array}{c}\text { Intensidade } \\
\text { de infestações }\end{array}$ \\
\hline Tabebuia ochracea & 5 & $5(100 \%)$ & 71 & $14,2 \pm 13,41$ \\
Melia azedarach & 70 & $23(32,9 \%)$ & 100 & $4,35 \pm 3,59$ \\
\hline
\end{tabular}


Rio 2002, Medel et al. 2004). Este tipo de distribuição espacial pode depender de fatores como o agrupamento dos hospedeiros, abundância dos hospedeiros e abundância e comportamento das aves dispersoras (López-de-Buen \& Ornelas 2002). O agrupamento dos hospedeiros poderia explicar a alta intensidade de parasitismo em Tabebuia ochracea, uma vez que essa espécie foi plantada no local e as árvores encontram-se próximas umas as outras, o que pode aumentar a conspicuidade do recurso para o dispersor, resultando consequentemente, num aumento nas taxas de infecção entre esses hospedeiros. Para Martínez-del-Rio et al. (1995) o fator primário que determina quantas sementes o hospedeiro vai receber é a presença de infecções prévias, sendo que um hospedeiro já parasitado provém frutos e poleiros acessíveis, portanto são mais utilizados do que potenciais hospedeiros que não se encontram parasitados (Roxburgh \& Nicolson 2005).

Nossos dados mostram que na área de estudo Phoradendron rubrum depende fortemente de Euphonia chlorotica e E. cyanocephala para dispersão de suas sementes, pois estas aves alimentam-se de grandes quantidades de frutos e são eficientes em depositar as sementes nos galhos das plantas hospedeiras. P. rubrum é encontrado parasitando Tabebuia ochracea e Melia azedarach e os níveis de infestações podem estar relacionados à distribuição espacial agregada e ao tamanho das espécies hospedeiras. No entanto, as diferenças encontradas também parecem estar fortemente relacionadas com o comportamento dos dispersores, seja por alimentarem-se preferencialmente em hospedeiros agrupados ou maiores. Portanto, tanto as características dos hospedeiros como o comportamento de forrageamento dos dispersores podem influenciar a presença e intensidade das infestações de P. rubrum na área de estudo.

Agradecimentos - Os autores agradecem a Mara Flávia Conti Nunes pela ajuda com as observações focais, ao Instituto Florestal pela permissão do trabalho na Floresta Estadual Edmundo Navarro de Andrade. E. Cazetta agradece à FAPESP (Proc. 00/14875-9), M. Galetti agradece a bolsa de produtividade do CNPQ. O LABIC recebe apoio da FAPESP, CNPq e Programa Cyted de Cooperação Internacional.

\section{Referências bibliográficas}

ARGEL-DE-OLIVEIRA, M.M. 1999. Frugivoria por aves em um fragmento de floresta de restinga no estado do Espírito Santo, Brasil. Tese de doutorado, Universidade Estadual de Campinas, Campinas.
AUKEMA, J.E. \& MARTÍNEZ-DEL-RIO, C. 2002. Where does a fruit-eating bird deposit a mistletoe seeds? Seed deposition patterns and an experiment. Ecology 83:3489-3496.

BANNISTER, P. \& STRONG, G.L. 2001. The distribution and population structure of the temperate mistletoe Ileostylus micranthus in the Northern Cemetery, Dunedin, New Zealand. New Zealand Journal of Botany 39:225-233.

CLAY, K., DEMENT, D. \& REYNANEK, M. 1985. Experimental evidence for host races in mistletoe (Phoradendron tomentosum). American Journal of Botany 72:1225-1231.

DOWNEY, P.O., GILL, A.M. \& BANKS, J.C.G. 1997. The influence of host attributes on mistletoe colonisation: an example from Mulligan's Flat Nature Reserve, ACT. Victorian Naturalist 114:105-111.

FORBES, W.A. 1880. On the structure of the stomach in certain genera of Tanagers. Proceedings of the Zoological Society 10:143-146.

GALETTI, M., PIZO, M.A. \& MORELLATO, L.P.C. 2002. Métodos para estudo de fenologia, frugivoria e dispersão de sementes. In Manual Brasileiro em Biologia da Conservação (L. Cullen Jr., R. Rudran \& C.V. Padua, eds.). Smithsonian Institution Press, Washington, p. 395-422.

GONDIM, M.J.C. 2001. Recurso alimentar para aves frugívoras em uma área de cerradão no estado de São Paulo. Tese de Doutorado, Universidade Estadual Paulista, Rio Claro.

JORDANO, P. 1992. Fruits and frugivory. In Seeds: the ecology of regeneration in plant communities (M. Fenner, ed.). C.A.B. International, Wallingford, p. 105-151.

KUIJT, J. 1969. The biology of parasitic flowering plants. University of California Press, Berkeley.

LADLEY,J.J.\& KELLY,D. 1996. Dispersal, germination and survival of New Zealand mistletoes (Loranthaceae): dependence on birds. New Zealand Journal of Ecology 20:69-79.

LÓPEZ-DE-BUEN, L. \& ORNELAS, J.F. 2002. Host compatibility of the cloud forest mistletoe Psittacanthus schiedeanus (Loranthaceae) in central Veracruz, México. American Journal of Botany 89:95-102.

MARTÍNEZ-DEL-RIO, C., HOURDEQUIN, M., SILVA, A. \& MEDEL, R. 1995. The influence of cactus size and previous infection on bird deposition of mistletoe seeds. Austral Journal of Ecology 20:571-576.

MARVIER, M.A. \& SMITH, D.L. 1997. Conservation implications of host use for rare parasitic plants. Conservation Biology 11:839-848.

MEDEL, R., VERGARA, E., SILVA, A. \& KALIN-ARROYO, M. 2004. Effects of vector behavior and host resistance on mistletoe aggregation. Ecology 85:120-126.

MONTEIRO, R.F., MARTINS, R.P. \& YAMAMOTO, K. 1992. Host specificity and seed dispersal of Psittacanthus robustus (Loranthaceae) in South-East Brazil. Journal of Tropical Ecology 8:307-314.

MOTTAJÚNIOR, J.C. 1991. A exploração de frutos como alimento por aves de mata ciliar numa região do distrito federal. Dissertação de mestrado, Universidade Estadual Paulista, Rio Claro. 
MOURA, L.C. 1998. Um estudo de estrutura de comunidades em fitocenoses originárias da exploração e abandono de plantios de eucalipto, localizadas no Horto Florestal Navarro de Andrade, Rio Claro (SP). Tese de doutorado, Universidade Estadual de Campinas, Campinas.

MURPHY, S.R., REID, N., YAN, Z. \& VENABLES, W.N. 1993 Differential passage time of mistletoe fruits through the gut of honeyeaters and flowerpeckers: effects on seedling establishment. Oecologia 93:171-176.

NORTON, D.A., LADLEY, J.J. \& OWEN, H.J. 1997. Distribution and population structure of the loranthaceous mistletoes Alepis flavida, Peraxilla colensoi and Peraxilla tetrapetala within two New Zealand Nothofagus forests. New Zealand Journal of Botany 35:323-336.

NORTON, D.A. \& CARPENTER, M.A. 1998. Mistletoes as parasites: host specificity and speciation. Trends in Ecology and Evolution 13:101-105.

NORTON, D.A. \& LADLEY, J.J. 1998. Establishment and early growth of Alepis flavida in relation to Nothofagus solandri branch size. New Zealand Journal of Botany 36:213-217.

OVERTON, J.M. 1994. Dispersal and infection in mistletoe metapopulations. Journal of Ecology 82:711-723.

OVERTON, J.M. 1996. Spatial autocorrelation and dispersal in mistletoes: field and simulation results. Vegetatio 125:83-98.

PIZO, M.A. 1997. Seed dispersal and predation in two populations of Cabralea canjerana (Meliaceae) in the Atlantic forest Southeastern Brazil. Journal of Tropical Ecology 13:559-577.

RADOMILJAC, A.M. 1998. The influence of pot host species, seedling age and supplementary nursery nutrition on Antalum album Linn. (Indian sandawood) plantation stablishment within the Ord River Irrigation Area, Western Australia. Forest Ecology and Management 102:193-201.

REID, N. 1989. Dispersal of mistletoes by honeyeaters and flowerpeckers: components of seed dispersal quality. Ecology 70:137-145.

REID, N. 1991. Coevolution of mistletoes and frugivorous birds. Australian Journal of Ecology 16:457-469.
REID, N., STAFFORD, M. \& YAN, Z. 1995. Ecology and population biology of mistletoes. In Forest Canopies (M.D. Lowman \& N.M. Nadkarni, eds.). Academic Press, New York, p. 285-311.

RESTREPO, C. 1987. Aspectos ecológicos de la diseminación de cinco especies de muérdagos por aves. Humbolditia 1:65-116.

RESTREPO, C., SARGENT, S., LEVEY, D.J. \& WATSON, D.M. 2002. The role of vertebrates in the diversification of New World mistletoes. In Seed Dispersal and Frugivory: Ecology, Evolution and Conservation (D.J. Levey, W.R. Silva \& M.Galetti, eds.). CABI Publishing, Wallingford, p. 83-98.

RIDLEY, R.S.\& TUDOR, G. 1989. The birds of South America. The Suboscine Passerines. University of Texas Press, Austin.

ROXBURGH, L. \& NICOLSON, W. 2005. Patterns of host use in two African mistletoes: the importance of mistletoehost compatibility and avian disperser behaviour. Functional Ecology 19:865-873.

SARGENT, S. 1995. Seed fate in a tropical mistletoe: the importance of host twig size. Functional Ecology 9:127-204.

SICK, H. 1997. Ornitologia Brasileira. Editora Nova Fronteira, Rio de Janeiro.

SNOW, B.K. \& SNOW, D.W. 1971. The feeding ecology of tanagers and honey creepers in Trinidad. The Auk 88: 291-322.

TENNAKOON, K.U. \& PATÊ, J.S. 1996. Effects of parasitism by a mistletoe on the structure and functioning of branches of its host. Plant Cell and Environment 19:517-528.

ULLMANN, I., LANGE, O.L., ZIEGLER, H., EHLERINGER, J., SCHULZE, E.D. \& COWAN, I.R. 1985. Diurnal courses of leaf conductance and transpiration of mistletoes and their hosts in Central Australia. Oecologia 67:577-587.

WILLIS, E.O. 2003. Birds of a eucalyptus woodlot in interior São Paulo. Brazilian Journal of Biology 63:141-158.

YAN, Z. \& REID, N. 1995. Mistletoe (Amyema miquelii and $A$. pendulum) seedling establishment on eucalypt hosts in Easter Australia. Journal of Applied Ecology 32:778-784.

ZAR, J.H. 1996. Biostatistical analysis. Prentice-Hall, New Jersey. 